\title{
CHEMICAL EFFECTS OF SHEAR ALFVÉN WAVES IN MOLECULAR CLOUDS
}

\author{
S.B. CHARNLEY ${ }^{1, \dagger}$ and W.G. ROBERGE ${ }^{2}$ \\ 1. Space Science Division, NASA Ames Research Center, California 94035. \\ 2. Physics Department, Rensselaer Polytechnic Institute, New York 12180.
}

\begin{abstract}
We consider the propagation of low-amplitude MHD waves in partially-ionised plasmas. Ion-neutral drift (ambipolar diffusion) can lead to significant variations in the deuterium fractionation ratios of several molecules (e.g. $\mathrm{HCO}^{+}$and $\mathrm{N}_{2} \mathrm{H}^{+}$) on spatial scales of between a few hundredths and a few tenths of a parsec, depending upon the fractional ionisation of the plasma. It is possible that interstellar Alfvén waves could be detected by molecular spectroscopy and that these waves may produce other small-scale abundance gradients in molecular clouds.
\end{abstract}

The dense interstellar medium consists of neutral molecular gas, ions, electrons, and charged grains. This partially-ionised plasma is threaded by a weak magnetic field $(\sim 1-40 \mu \mathrm{G})$ to which the ions, electrons and the negatively charged dust grains are attached; the plasma and dust are collisionally coupled to the neutral component. Perturbations of this system can generate a spectrum of magnetohydrodynamic (MHD) waves of which, for molecular clouds, the two most important types are compressional (magnetosonic) waves and shear Alfvén waves (Zweibel \& Josafatsson 1983).

We have explored the chemistry of molecular gas in which shear Alfvén waves propagate. The magnetohydrodynamical structure and evolution of the wave packet are governed by Maxwell's equations, plus dynamical equations for the coupled fluids of neutral particles, ions, electrons, and dust grains (e.g. Elmegreen 1979; Draine 1986). Solution of the linearised problem (Roberge \& Hanany 1991) indicates that appreciable ion-neutral drift speeds, $v_{n i}$, can occur $\left(v_{n i}<1 \mathrm{~km}\right.$ $\left.\mathrm{s}^{-1}\right)$. Charnley \& Roberge (1991a) have pointed out that this may have important consequences for interstellar chemistry: several important endothermic ion-molecule reactions may proceed rapidly in such a wave. Enhanced rates for reaction of $\mathrm{H}_{2}$ with $\mathrm{H}_{2} \mathrm{D}^{+}, \mathrm{CH}_{2} \mathrm{D}^{+}$and $\mathrm{C}_{2} \mathrm{HD}^{+}$, suggest that the high degree of deuterium fractionation observed in cold molecular clouds may offer a test for the chemical signature of Alfvén waves. An important quantity for the time-dependent ion-molecule chemistry is the time-scale of the coupling of the neutrals to the wave by elastic collisions with the streaming ions, $\tau_{n i}$, which is inversely proportional to the fractional ionisation, $x_{e}$. A related quantity is the slowing-down length, $L_{n i}=\tau_{n i} v_{A}$, where $v_{A}$ is the Alfven speed. If the characteristic time-scale for a given chemical reaction to occur is $\tau_{R}$, it will be affected by the presence of a wave if the ratio $\tau_{n i} / \tau_{R}$ is (much) greater than unity. The maximum value of $v_{n i}$ determines the most endothermic reaction that can be driven efficiently in the wave.

For isothermal shear Alfvén waves propagating in cold gas the only effect which can alter the molecular chemistry is the kinetic energy contributed to ion-neutral processes by ambipolar diffusion. We have incorporated this effect in a similar manner to that used to study the destruction of deuterated molecules in C-type MHD shocks (Pineau des Foréts et al. 1989). The deuterium chemistry model evolves in a 'pseudo-time-dependent' manner (Millar et al. 1989) until some time, $t_{\text {wave }}$, when the parcel of gas is swept by an Alfven wave; the chemical evolution is then followed for times $\sim 5 \tau_{n i}$. Figure 1 shows the chemistry in one representative model for $t_{w a v e}=10^{5}$ years, at which point the chemistry is well-developed and a significant degree of $\mathrm{D}$ fractionation has occurred.

†NAS/NRC Resident Research Associate 


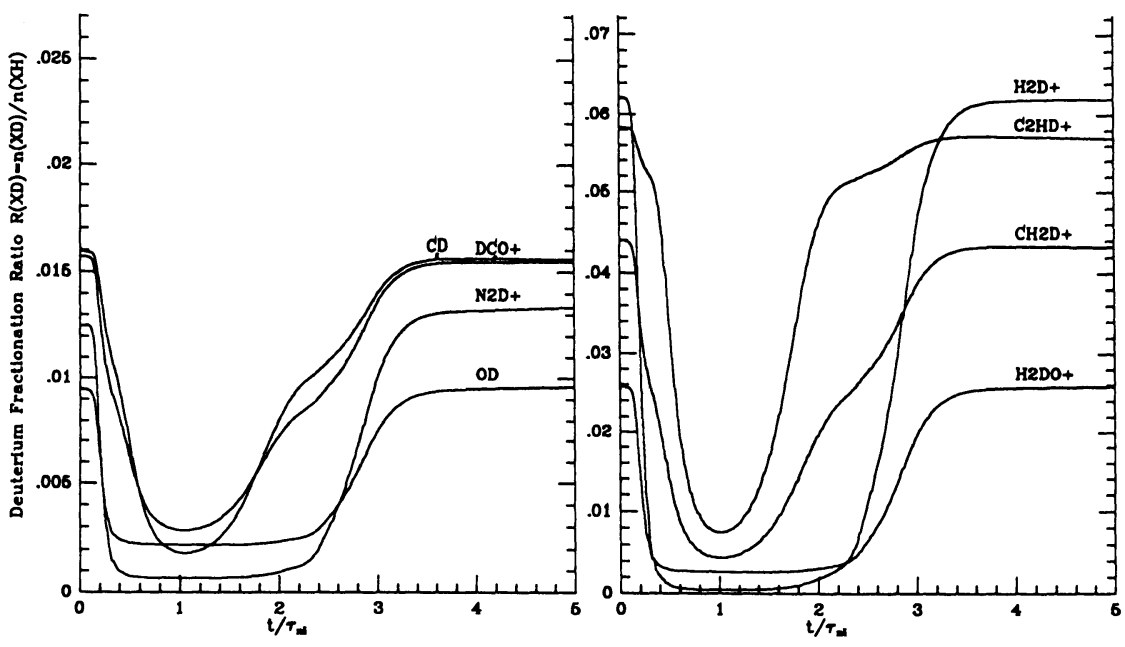

Figure 1. Evolution of $D$ fractionation ratios in an Alfvén wave propagating in gas with $n_{H}=2 \times 10^{4} \mathrm{~cm}^{-3}, T_{k i n}=12 \mathrm{~K}$, and $x_{e}=1.7 \times 10^{-7}$. For this model $\tau_{n i}=5.2 \times 10^{3}$ years, $L_{n i}=$ $0.01 \mathrm{pc}$, and the peak value of $v_{n i}$ is $1.05 \mathrm{~km} \mathrm{~s}^{-1}$.

As $\mathrm{H}_{2} \mathrm{D}^{+}$and $\mathrm{H}_{3}^{+}$are difficult to detect in the ISM, the species which appear to be the best candidates for diagnostics of MHD wave propagation are $\mathrm{DCO}^{+}$and $\mathrm{N}_{2} \mathrm{D}^{+}$. Depending upon the ion-neutral drift speed $\left(v_{n i} \sim 0.5-1 \mathrm{~km} \mathrm{~s}^{-1}\right)$ and the fractional ionisation of the gas, $R\left(\mathrm{DCO}^{+}\right)$ and $R\left(\mathrm{~N}_{2} \mathrm{D}^{+}\right)$can easily vary by factors of between 3 and 10 over spatial scales of $\sim 0.01-0.1 \mathrm{pc}$ (Charnley \& Roberge 1991b). High resolution mapping of dark clouds may discover the predicted variation in deuterium fractionation ratios and could allow the detection of hydromagnetic waves by molecular spectroscopy. We note that published maps of TMC-1 do show spatial variations in the emission of $\mathrm{DCO}^{+}$and $\mathrm{HCO}^{+}($Guélin et al. 1982). The theory predicts that the molecular gas with the narrowest linewidths should contain the highest values of $R(\mathrm{XD})$; observations by Bell et al. (1988) of $R\left(\mathrm{C}_{3} \mathrm{HD}\right)$ in several dark clouds clearly show this trend.

We have developed this simple model to study the deuteration effects of one shear Alfvén wave, however, other ion-molecule reactions with small activation barriers may also be driven by these waves, and this work suggests that the presence of small-scale abundance gradients in dark clouds is related to the local level of MHD turbulence. MHD turbulence will generally act to produce spatial magnetic field gradients within interstellar clouds and the resulting ambipolar diffusion can provide a comprehensive mechanism to drive endothermic ion-molecule reactions.

\section{References}

Bell, M.B. et al. 1988, Ap.J., 326, 924.

Charnley, S.B. \& Roberge, W.G. 1991a, in Molecular Clouds,

eds. R.A. James \& T.J. Millar, Cambridge, p.247

Charnley, S.B. \& Roberge, W.G. 1991b, in preparation.

Draine, B.T. 1986, M.N.R.A.S., 220, 133.

Elmegreen, B.G. 1979, Ap.J., 232, 729.

Guélin, M., Langer, W.D. \& Wilson, R.W. 1982, Astr.Ap., 107, 107.

Millar, T.J., Bennett, A. and Herbst, E. 1989, Ap.J., 340, 906.

Pineau des Forêts, G., Roueff, E. \& Flower, D. 1989, M.N.R.A.S., 240, 167.

Roberge, W.G. \& Hanany, S. 1991, Ap.J., , submitted.

Zweibel, E.G. \& Josafatsson, K. 1983, Ap.J., 270, 511. 